FORMATION Formation emploi

Revue française de sciences sociales

143 | Juillet-Septembre 2018

Le retour en formation : une vraie chance?

\title{
L'accompagnement des jeunes en situation de décrochage scolaire : inégalités et non-recours
}

Supporting early school leavers : inequalities and non-use

Begleitung junger Schulabbrecher: Ungleichheit und ungenutzte Möglichkeiten

El acompañamiento de los jóvenes en situación de abandono escolar :

desigualdades y no utilización

Pierre-Yves Bernard

\section{OpenEdition}

Journals

Édition électronique

URL : http://journals.openedition.org/formationemploi/6103

DOI : 10.4000/formationemploi.6103

ISSN : 2107-0946

Éditeur

La Documentation française

Édition imprimée

Date de publication : 20 novembre 2018

Pagination : 33-55

ISSN : 0759-6340

Référence électronique

Pierre-Yves Bernard, «L'accompagnement des jeunes en situation de décrochage scolaire : inégalités et non-recours », Formation emploi [En ligne], 143 | Juillet-Septembre 2018, mis en ligne le 20 novembre 2018, consulté le 05 novembre 2020. URL : http://journals.openedition.org/ formationemploi/6103 ; DOI : https://doi.org/10.4000/formationemploi.6103 


\title{
L'accompagnement des jeunes en situation de décrochage scolaire : inégalités et non-recours
}

\author{
Pierre-Yves Bernard \\ Chercheur en sciences de l'éducation, Centre de recherche en éducation de Nantes
}

Résumé

L'accompagnement des jeunes en situation de décrochage scolaire : inégalités et non-recours

Les politiques de remédiation du décrochage scolaire s'appuient sur des dispositifs d'accompagnement visant au raccrochage en formation et/ou à l'insertion professionnelle. Pourtant, un non-recours important à ces dispositifs interroge sur la capacité des bénéficiaires potentiels à y accéder. Une étude réalisée auprès d'un échantillon de 2948 jeunes repérés en situation de décrochage scolaire révèle d'importantes inégalités territoriales dans leur prise en charge, ainsi que l'effet cumulé des insuffisances de l'offre d'accompagnement et de l'inadéquation des propositions faites aux jeunes dans la construction de ces inégalités.

Mots clés : abandon des études, mesure jeune, accompagnement en formation, accompagnement professionnel, disparité régionale, jeune en difficulté, non-recours

Abstract

Supporting early school leavers: inequalities and non-use

The policies of early school leaving compensation are based on support measures in order to facilitate access to training or employment. However, a lot of beneficiaries don't use these measures, that question their ability to access them. A study based on a sample of 2948 young people identified as early school leavers shows strong territorial inequalities in mentoring, and the cumulative effects of the lack of support supply and of the unfit proposals in the building of these inequalities.

Keywords: drop out, youth employment scheme, coaching in training, coaching, regional disparity; young person in difficulty, non-take-up

Journal of Economic Literature: I 21 ; I 28

Traduction : Auteur. 
Le décrochage scolaire est l'objet de différents niveaux d'action publique, à savoir la prévention, l'intervention, la compensation. Les deux derniers volets ont été particulièrement développés dans le contexte français, notamment dans le cadre des politiques d'insertion sociale et professionnelle des jeunes (Denantes, 2008). Pourtant, un temps de latence parfois long sépare la rupture scolaire de la prise en charge des jeunes non diplômés par les dispositifs d'accompagnement (missions de lutte contre le décrochage scolaire, missions locales, etc.) visant à les mener à la qualification (Berthet $\&$ Zaffran, 2014).

On se propose ici d'examiner l'écart entre, d'une part, l'engagement institutionnel visant à "trouver une solution pour chaque jeune " et, d'autre part, les formes de non-recours aux dispositifs d'accompagnement. L'objectif est de mieux comprendre comment se construit ou non le recours à ces dispositifs.

Le recours aux dispositifs d'accompagnement est considéré ici comme une séquence allant du repérage des jeunes en situation de décrochage, jusqu’à leur enrôlement dans un dispositif. Ce cadre d'analyse permet de réaliser une étude quantitative des facteurs de recours à chaque étape du processus.

Pour ce faire, nous exploitons les résultats d'une enquête réalisée auprès de jeunes repérés comme "décrocheurs" $(\mathrm{N}=2$ 948). Interrogés environ un an en moyenne après leur rupture scolaire, ils déclarent les contacts qu'ils ont pu avoir ou non avec des professionnels dans les premiers mois de leur parcours post-scolaire, et les actions qui en ont éventuellement découlé ${ }^{1}$.

Ce travail permet de tester deux hypothèses, associées elles-mêmes à deux modélisations différentes du non-recours (Warin, 2012). La première se situe du côté des institutions en charge de l'accompagnement des jeunes en difficulté. Elle explique le non-recours par l'insuffisance de l'offre d'accompagnement, tant en matière d'informations auprès des bénéficiaires potentiels qu'en termes de personnel et de dispositifs accessibles sur le territoire où vivent ces bénéficiaires. La seconde hypothèse se situe au niveau des interactions entre les jeunes et les agents représentant ces institutions. Elle met en avant la "non-demande ", au sens où le non-recours exprime une forme de remise en cause de la politique publique par les bénéficiaires eux-mêmes, révélatrice en l'espèce de l'inadéquation de cette politique aux attentes d'une partie de la jeunesse.

Dans une première partie, on revient sur la place de l'accompagnement dans les politiques de lutte contre le décrochage scolaire. Une deuxième partie propose une approche séquentielle des pratiques d'accompagnement. Une troisième partie est consacrée à la

1. Elles constituent le matériau d'un programme de recherche financé par l'Agence nationale de la recherche, intitulé "Territoires et décrochages scolaires " (n ANR-14-CE30-0009-01), coordonné par Pierre-Yves Bernard, et qui regroupe une vingtaine de chercheurs de quatre laboratoires (Centre de recherche en éducation de Nantes, Espaces et société de Caen, Centre Émile Durkheim de Bordeaux, Laboratoire d'économie et de sociologie du travail d'Aix-en-Provence). 
présentation des données d'enquête utilisées dans cette recherche. Enfin la dernière partie présente les résultats des analyses réalisées. Ils mettent en évidence de fortes inégalités d'accès aux dispositifs d'accompagnement selon les territoires.

\section{Accompagnement, latence et non-recours après le décrochage scolaire}

Bien qu'une offre diversifiée d'accompagnement soit proposée aux jeunes en situation de décrochage scolaire, tous n'en bénéficient pas. Ce non-recours est habituellement interprété en termes de repérage des jeunes par les dispositifs en charge de la lutte contre le décrochage.

\subsection{Une offre diversifiée d'accompagnement}

Les pratiques d'accompagnement occupent une place importante dans les politiques de lutte contre le décrochage scolaire. Celles-ci peuvent être déclinées selon qu'elles sont plus ou moins préventives ou réparatrices (Bernard, 2015). Dans tous les cas, une attention particulière est accordée à l'individualisation des actions à entreprendre, afin de permettre la persévérance scolaire ou le raccrochage en formation d'enfants ou de jeunes en difficulté scolaire. Couplée avec la nécessité de placer le jeune en situation d'acteur de ses apprentissages, l'individualisation oriente l'activité des professionnels dans le sens de l'aide et de l'accompagnement. Ainsi, les pratiques de prévention du décrochage scolaire développées en Europe "mettent l'accent sur l'apport d'une aide personnalisée aux élèves" (Eurydice-Cedefop, 2014, p. 60).

La question de l'accompagnement est encore plus saillante au moment où les premiers signes de décrochage apparaissent, et notamment l'absentéisme. En France, c'est le sens des interventions des dispositifs relais au collège (Benhaim-Grosse, 2012), ou des actions mises en place dans le cadre des Missions de lutte contre le décrochage scolaire $(\mathrm{MLDS})^{2}$ dans le second cycle du second degré. Ces dernières proposent des actions de remédiation et d'accès à la qualification, où l'accompagnement personnalisé occupe une place importante (Bernard \& Michaut, 2006, 2013).

Quand les élèves ont quitté l'école sans diplôme, ils constituent la cible des politiques de compensation du décrochage scolaire. La pratique de l'accompagnement vise alors à permettre le retour en formation, l'insertion sociale et professionnelle, ou plus largement de trouver des solutions aux difficultés des jeunes sortis tôt du système éducatif et

2. Les MLDS ont succédé aux MGI (Missions générales d'insertion) en 2013. Les pratiques, mesures et personnels restent cependant très stables malgré le changement de sigle. 
dépourvus le plus souvent des ressources sociales leur permettant d'affronter de manière autonome l'entrée dans la vie adulte.

Ces pratiques se sont structurées historiquement à partir de la problématique de l'insertion des jeunes peu qualifiés, particulièrement développées en France autour du réseau des Missions locales (Castra, 2006). Plus largement, l'accompagnement désigne un vaste ensemble de pratiques hétérogènes, assez fortement différenciées selon le degré d'autonomie dévolu à l'accompagné et le pouvoir exercé par l'accompagnateur, et qui traduit les transformations des sociétés contemporaines dans le sens d'une plus grande individualisation des rapports sociaux (Paul, 2009 ; Demailly, 2009).

Dans les champs de l'éducation et de l'insertion, ces pratiques sont portées par des professionnels de provenances diverses, à savoir enseignants, conseillers d'orientation, éducateurs spécialisés, conseillers d'insertion, rattachés à des institutions elles-mêmes diverses, comme les établissements scolaires, les associations, les collectivités territoriales, etc. Cela nécessite un travail de collaboration interinstitutionnelle important et incertain (White \& Wehlage, 1995 ; Bernard \& Michaut, 2014a).

\subsection{Le non-recours à l'accompagnement ou à la seconde chance}

Pour autant, les jeunes sortant sans diplôme du système éducatif ne bénéficient pas nécessairement des dispositifs d'accompagnement qui leur sont destinés, tout au moins dans les premiers mois qui suivent leur rupture scolaire. En 2008, un rapport du CERC (Conseil de l'emploi, des revenus et de la cohésion sociale) notait le décalage important entre le nombre de jeunes pris en charge par les MGI (Missions générales d'insertion), assez restreint, et le nombre bien plus important de sortants sans diplôme tel qu'il était estimé par l'enquête Emploi. Pour Bernard (2009), à partir d'une étude sur la région des Pays-de-la-Loire, on pouvait estimer que sur la période 2000-2005, 70 à $80 \%$ des jeunes en situation de décrochage scolaire n'étaient pas pris en charge par la MGI.

Cette faiblesse contraste fortement avec l'importance des prises en charge par les Missions locales (De Foucauld \& al., 2010). Toutefois, celles-ci interviennent tardivement dans le parcours des jeunes sans diplôme. Ainsi, une étude réalisée sur la région Rhône-Alpes estimait à 28 mois le temps de latence entre la rupture scolaire et la prise en charge par les Missions locales (PRAO, 2013).

Il y a donc un véritable non-recours en la matière, au sens où des personnes éligibles à une offre d'action publique n'en bénéficient pas. Cette problématique du non-recours, née dans le champ des politiques de prestations sociales, s'est déployée sur l'ensemble des politiques publiques visant des usagers, dans un contexte d'interrogation sur l'effectivité et la pertinence de ces politiques (Warin, 2003 ; op. cit.).

Selon Warin, le non-recours recouvre trois situations que sont la non-connaissance, lorsque l'offre publique n'est pas connue par les bénéficiaires, la non-demande, quand 
elle est connue, mais non demandée, la non-réception, quand elle est connue, demandée, mais pas obtenue (2012).

Cette approche permet de dépasser une vision triviale du non-recours qui serait due à une simple méconnaissance des droits, pour envisager la possibilité que l'offre publique puisse ne pas correspondre aux attentes des usagers, ou encore qu'elle sélectionne ceuxci à partir de critères qui maximisent l'efficacité de l'offre publique, au détriment des publics les plus défavorisés socialement. L'évaluation des dispositifs d'insertion a mis en évidence depuis longtemps ces phénomènes de sélection, qui affectent particulièrement les actions d'accompagnement (Mas, 2002 ; Darmon, Frade, Demazière \& Haas, 2004).

La réussite d'un programme d'accompagnement repose en effet sur la co-construction du projet et du parcours entre la personne accompagnée et le professionnel chargé de l'accompagnement (Rouleau-Berger, 1998), et donc sur des compétences que la personne accompagnée peut révéler à travers les éléments de son parcours scolaire antérieur, fut-il incomplet. Ainsi, Bernard \& Michaut (op. cit.) montrent que les actions d'accompagnement de la MLDS sont d'autant plus efficaces que le décrochage scolaire est intervenu tardivement dans le parcours scolaire du jeune, et donc qu'en la matière, l'action publique bénéficie davantage aux moins défavorisés. Ce type de résultat va dans le sens d'une prise en compte des interactions entre jeunes et professionnels, qui sont consubstantielles à la pratique de l'accompagnement. Une partie de ces interactions vise à ajuster attentes des jeunes et propositions institutionnelles, notamment en orientant les demandes des jeunes vers des projets "réalistes " et les secteurs d'activité susceptibles de les accueillir (Zunigo, 2008).

La réussite de ces interactions, au regard des critères institutionnels d'efficacité, amène donc les dispositifs à sélectionner les publics en fonction d'indicateurs de motivation qui sont souvent l'expression de compétences sociales inégalement réparties (Denecheau, Houdeville, Mazaud, 2015).

\subsection{Des institutions pro-actives?}

Une autre spécificité de l'action publique en direction des jeunes en décrochage scolaire est la responsabilité donnée a priori à l'institution dans l'initiative d'offrir une aide. Il ne s'agit pas seulement de faire en sorte que des usagers puissent accéder à des droits, mais de solliciter les jeunes ayant droit à un accompagnement, à partir de critères les définissant comme " décrocheurs ". Dès lors, la question du repérage des personnes concernées est particulièrement cruciale. Le non-recours par non-connaissance ne repose pas seulement sur l'absence d'information dont disposeraient les jeunes, mais également sur l'absence de prise de contacts à l'initiative d'institutions, scolaires ou éducatives. Par exemple, Plessard, Simon \& Berthet (2014), à partir d'un échantillon de jeunes accueillis en Mission locale dans la région Rhône-Alpes, montrent que la moitié environ 
des jeunes déclare ne pas avoir reçu de proposition d'aide pendant cette période de latence, alors que, pour la majorité d'entre eux, ils auraient souhaité en obtenir.

Cette question du non-recours amène les politiques éducatives à se doter d'outils permettant d'identifier les jeunes en décrochage. Dans la plupart des pays d'Europe, les autorités éducatives ont mis en œuvre, ces dernières années, des outils de repérage des situations de décrochage (Eurydice, op. cit., p. 29). En France, depuis 2011, un Système interministériel d'échange d'informations (SIEI) permet d'identifier les jeunes ayant interrompu une formation secondaire sans avoir obtenu de diplôme. Sur la base des renseignements fournis par SIEI, les acteurs de terrain, réunis dans les Plateformes de suivi et d'accueil des décrocheurs (PSAD), sont chargés de contacter les jeunes sans solution de formation, afin de leur proposer un accompagnement vers la formation et l'insertion. Ce type d'outil suppose la construction de partenariats entre institutions différentes (en l'occurrence le ministère de l'Éducation nationale, le ministère de l'Agriculture au titre de l'enseignement agricole, les Régions au titre de l'apprentissage, le ministère de la Justice au titre de la Protection judiciaire de la jeunesse, etc.).

\section{Une approche séquentielle du recours à l'accompagnement}

Un consensus peut être dégagé des travaux sur le non-recours : celui-ci se construit en plusieurs étapes. Dans le cas de l'accompagnement des jeunes en situation de décrochage scolaire, certaines de ces étapes sont spécifiques (repérage et prise de contact), alors que d'autres ont été mises en évidence dans d'autres contextes des politiques sociales (proposition d'aide, enrôlement).

\subsection{Le non-recours, un processus en plusieurs étapes}

Comme le suggèrent les distinctions opérées par Warin, évoquées précédemment, le non-recours peut être analysé comme le résultat d'un processus en plusieurs étapes. Pour recourir à une offre publique, il faut d'abord en avoir connaissance, puis en faire la demande, et enfin obtenir la prestation demandée.

D'autres travaux présentent une version plus complexe de ces étapes. Ainsi, Heckman \& Smith (2003) distinguent cinq étapes dans l'accès à un programme américain d'accompagnement de personnes désavantagées (faibles revenus, bénéficiaires d'aides sociales, faible niveau d'anglais, etc.) pour accéder à l'emploi : éligibilité, information, candidature, acceptation et participation. En s'inspirant de ce modèle, Dmitrijeva, Fremigacci \& l'Horty (2015) analysent le recours à l'accompagnement des bénéficiaires du Revenu social d'activité (RSA), en France en trois étapes : l'invitation à participer, l'acceptation de l'invitation et la présence à l'invitation. 
L'intérêt de ces analyses est de montrer que le recours (et donc le non-recours) est le résultat cumulatif d'un certain nombre de séquences, d'où le qualificatif de séquentiel que nous proposons ici. En distinguant ces séquences, il est possible de concevoir le non-recours comme le produit des probabilités de ne pas satisfaire aux différentes étapes successives de l'accès à l'offre publique.

De ce fait, il est possible de considérer les différents facteurs, individuels et contextuels, qui déterminent cette probabilité pour chaque séquence. Par exemple, de faibles ressources sociales et culturelles peuvent constituer un frein pour accéder à la connaissance d'un dispositif, alors que l'existence de ressources sociales alternatives engage certaines personnes à refuser un dispositif, même s'ils en ont eu connaissance. Appréhender le non-recours dans sa globalité amènerait alors à considérer ces différents facteurs comme agissant simultanément, alors qu'en fait ils se rapportent à des séquences différentes du processus d'accès au dispositif.

L'intérêt de cette approche ne réside pas seulement dans l'amélioration de la compréhension des facteurs de recours ou de non-recours. Cette analyse s'inscrit aussi dans une démarche théorique qui considère simultanément les logiques d'acteurs et les cadres institutionnels, dans ce qu'ils les autorisent ou les contraignent. Les acteurs ne sont pas déterminés par le contexte, ils agissent en fonction des ressources auxquelles ils peuvent avoir réellement accès, en s'appuyant sur des logiques d'action construites par l'expérience.

De ce point de vue, le contexte institutionnel peut augmenter leur capacité d'action, comme il peut la limiter. Une analyse en situation permet de mettre à jour ce qui relève de ressources institutionnelles, d'une part, et ce qui relève des usages et des non-usages de ces ressources, d'autre part, en portant l'attention sur la façon dont la relation de l'acteur à l'institution se construit dans un ensemble d'interactions.

L'approche séquentielle vise alors à "styliser » les faits, selon l'expression des économistes, afin de dégager les principales étapes de ces interactions. L'objectif n'est pas ici de reconstruire l'ensemble des processus inscrits dans les biographies des individus, mais de se limiter aux séquences spécifiques au recours ou au non-recours à l'accompagnement en situation de décrochage.

Le parcours qui précède le décrochage est malgré tout important. Ainsi, les jeunes qui sont susceptibles de bénéficier de mesures de prévention ou de remédiation du décrochage ont déjà une expérience scolaire, généralement très négative (Bernard \& Michaut, 2014b). L'analyse doit prendre en compte cette expérience, dans la mesure où elle affecte la logique d'action aux différentes étapes du parcours qui suit le décrochage scolaire. 


\subsection{Une approche en quatre temps}

Lapproche séquentielle proposée ici se structure en quatre temps. Les séquences choisies ici sont en partie spécifiques aux questions d'accompagnement des jeunes en situation de décrochage. En effet, les modèles séquentiels proposés par la littérature sont plutôt construits à partir des problématiques des politiques sociales. Bien qu'ils aient une valeur heuristique importante, il n'est pas possible de les appliquer tels quels ici. Deux étapes sont en effet spécifiques en l'espèce.

La première, présentée dans la partie précédente, est constituée par le repérage des jeunes décrocheurs. Il est hautement probable que le repérage effectué par les institutions éducatives ou d'insertion ne couvre pas l'ensemble des personnes en décrochage scolaire. Labsence de repérage peut provenir de causes institutionnelles, quand par exemple les établissements scolaires ne déclarent pas des décrochages en cours d'année, afin de ne pas être considérés comme des établissements en difficulté auprès des autorités éducatives. Des jeunes en situation de décrochage scolaire peuvent donc ne pas être repérés, et de ce fait ne pas recevoir de proposition d'aide. Toutefois, l'analyse de cette première séquence du non-recours est particulièrement délicate. Par définition, les jeunes non repérés ne sont pas connus, et il n'est pas possible de comparer, au niveau individuel, la population des jeunes repérés avec une population de référence. Les écarts entre repérage et situations effectives ne peuvent être perçus qu’à travers la comparaison des données administratives issues de ce repérage avec d'autres données d'enquêtes ou de recensements. Mais on ne peut pas caractériser ces écarts en termes de variables individuelles. Il est cependant envisageable de dégager quelques enseignements en comparant les structures de ces données, en fonction de leurs caractéristiques communes.

La deuxième étape spécifique est celle de la prise de contact. Rappelons qu'il est généralement de la responsabilité des acteurs du système éducatif de contacter les jeunes afin de leur proposer une solution. Ce sont les PSAD qui assument cette fonction. Or, même si les textes réglementaires indiquent que chaque personne repérée doit se voir proposer une solution d'accompagnement, il est tout à fait possible que toutes les personnes en droit d'être contactées ne le soient pas effectivement. D'une part, les données permettant ce contact peuvent ne plus être valides (changements d'adresse, ou, plus fréquent, changements de numéros de téléphone); d'autre part, le nombre de personnes à contacter peut surpasser les capacités dont se sont dotées les institutions chargées de ces prises de contact. Un jeune repéré peut donc ne pas avoir été contacté, et de fait ne pas avoir la possibilité de recevoir une aide à laquelle il a pourtant droit. Si les bases administratives utilisées par les institutions indiquent le suivi et les prises de contact, il est alors possible de mener une analyse de ce niveau de non-recours en fonction des caractéristiques figurant dans ces bases.

Si un jeune en situation de décrochage est repéré, et qu'il est contacté, il entre alors dans une série d'étapes classiquement distinguées dans la littérature portant sur le non-recours. On propose ici de les représenter par deux séquences principales. D’une part, celle de la proposition d'aide. Il est en effet important de la distinguer de la précédente. La prise de 
contact ne constitue pas nécessairement en soi une proposition d'aide. Elle peut se manifester, par exemple, par la proposition d'un rendez-vous pour un entretien, ce que le jeune ne perçoit pas forcément comme une aide. Cette séquence est fortement caractérisée par une suite d'interactions entre le jeune éligible et l'institution, interactions où les jugements portés par les personnes sur les situations sont déterminants, au sens où ils qualifient (ou disqualifient) une éventuelle relation d'accompagnement. Pour reprendre l'exemple précédent, un jeune qui se rend à un rendez-vous dans un centre d'information et d'orientation peut considérer qu'il n'y a eu aucune proposition d'aide, si dans la situation d'un premier contact, un conseiller d'orientation organise un entretien assez informel visant à tester ses motivations. Inversement un professionnel de l'orientation peut considérer qu'il ne peut faire aucune proposition à un jeune, jugeant que celui-ci doit régler des problèmes qui ne sont pas de son ressort, par exemple des problèmes d'addiction. Ce sont bien les jugements en situation qui définissent ici le recours (ou le non-recours), jugement qui s'appuie à la fois sur des attentes du côté du jeune, et sur des conceptions de l'activité professionnelle du côté des agents des institutions.

Quand les jugements des personnes s'accordent sur l'existence d'une proposition d'aide, c'est-à-dire quand le professionnel fait une proposition qui est reconnue comme telle par le jeune en situation de décrochage scolaire, une deuxième séquence s'amorce, que l'on désignera comme celle de l'enrôlement. La relation d'accompagnement est alors finalisée vers un objectif (accès à une formation, un logement, etc.) correspondant à la fois à l'offre institutionnelle et aux attentes du bénéficiaire.

\section{Des données administratives 3 qui renseignent sur la prise de contact}

Les données exploitées sont principalement les fichiers SIEI de cinq académies, et les résultats d'une enquête téléphonique à partir de ces fichiers.

Les fichiers SIEI sont issus des recensements effectués deux fois par an. Ils visent à identifier de manière exhaustive les jeunes de plus de 16 ans ayant interrompu leur formation secondaire sans avoir obtenu le diplôme terminal correspondant à cette formation. Plus précisément, les jeunes " décrocheurs" sont définis de la manière suivante. Ainsi, ils doivent:

- avoir été scolarisés au moins 15 jours en continu au cours de l'année scolaire en cours ou de l'année scolaire précédente ;

- être âgés de 16 ans au moins ;

- ne pas avoir atteint le niveau de diplôme fixé par voie règlementaire ;

- ne plus être inscrits dans un système de formation initiale (Éducation nationale, Agriculture, Centres de formation d'apprentis (CFA)). 
Les fichiers utilisés sont issus du recensement de novembre 2014. Toutefois, il ne s'agit pas des fichiers d'origine, mais de leur état en mars 2015, après plusieurs mois d'utilisation par les professionnels des PSAD. Ce point est particulièrement important, puisque les données disponibles permettent d'avoir une évaluation des personnes contactées par ces professionnels, et renseignent donc particulièrement sur l'étape " prise de contact " du processus d'accompagnement.

\section{Encadré 1. L'enquête}

Les cinq académies ont été choisies pour leur caractère contrasté. Sans prétendre à une représentativité au niveau national, elles présentent une diversité de situations au niveau de l'ampleur du décrochage scolaire. Elles représentent le niveau institutionnel de notre étude. La lutte contre le décrochage scolaire est en effet déconcentrée au niveau académique, depuis les années 1990. Bien que la mise en place du SIEI (Système interministériel d'échange d'informations), en 2011, représente une forme de recentralisation de cette politique, il nous a semblé pertinent d'observer l'offre de repérage et de remédiation au niveau académique. En effet, la politique de lutte contre le décrochage scolaire relève en partie des compétences rectorales de l'administration de l'éducation nationale, notamment par les Missions de lutte contre le décrochage scolaire, organisées de manière différente selon les académies (Bernard, op. cit.).

À partir de ces fichiers, une enquête téléphonique a été réalisée auprès des jeunes y figurant, entre avril et octobre 2015. II s'agit d'une enquête MODS (Motifs de décrochage scolaire), à partir des indicateurs retenus par Bernard \& Michaut $(2014 b, 2016)$. Dans la suite du texte, cette enquête sera appelée MODS 2015.

Le questionnaire comporte cinq parties. La première reprend les données du fichier SIEI, notamment la dernière formation suivie et la date d'interruption (signalement) des études. La deuxième porte sur l'expérience scolaire (éventuels redoublements, type de troisième fréquentée, choix d'orientation, rapports avec les enseignants, etc.). La troisième partie est consacrée aux motifs auto-déclarés de décrochage scolaire des jeunes, à partir d'une batterie d'items sur lesquels les enquêtés se positionnent. La quatrième partie regroupe les questions portant sur la situation des jeunes au moment de l'enquête, et sur l'éventuel accompagnement dont ils ont pu bénéficier. La dernière partie fournit les caractéristiques sociologiques déclarées par les jeunes.

Le grand nombre de personnes figurant dans le fichier n'autorisait pas une exploration exhaustive de la population. Un échantillon a donc été constitué $(\mathrm{N}=7199)$ afin de déterminer les personnes à contacter par les enquêteurs et les enquêtrices, la représentativité de l'échantillon étant contrôlée selon le sexe, le type d'établissement fréquenté au moment du décrochage, et la localisation géographique (niveau département).

Sur cet échantillon, beaucoup de personnes n'ont pu être jointes, notamment en raison de coordonnées inexactes dans le fichier d'origine. Plus surprenant, un grand nombre de personnes contactées ont déclaré n'avoir jamais interrompu leurs études, ce qui les plaçait alors de facto en dehors du champ de l'enquête. Compte tenu des refus de réponse et des questionnaires incomplets, on a pu interroger 2948 jeunes dans les cinq académies. Cet échantillon ne comporte que des jeunes ayant déclaré avoir décroché au sens de SIEI. Pour s'assurer de sa représentativité, il a été redressé en fonction des variables disponibles du fichier SIEl d'origine : académie, âge, sexe et niveau de formation. 


\section{Du repérage à l'enrôlement : les déterminants du recours à l'accompagnement}

\subsection{Des inégalités territoriales dans le repérage du décrochage scolaire}

Il n'est pas possible d'évaluer directement les inégalités de repérage des publics susceptibles de bénéficier de l'action publique. On peut toutefois comparer des données de recensement de population avec les données de SIEI. Les premières nous renseignent sur la part des non-diplômés en pourcentage d'une classe d'âge, par exemple les 16-24 ans. Ce sont des données de stock à une date donnée. Les données SIEI, quant à elles, renseignent sur les entrées en décrochage, quel que soit l'âge. Ce sont des données de flux. Soulignons que ces données ne sont pas strictement comparables. SIEI enregistre en effet des diplômés comme décrocheurs. Ce sont les titulaires d'un diplôme du secondaire (par exemple un CAP - certificat d'aptitude professionnelle) qui décrochent lors d'une formation ultérieure, par exemple en bac pro. Ces personnes ne sont pas considérées comme non diplômées au sens du recensement.

Que nous enseigne une comparaison entre académies ? On peut raisonnablement faire l'hypothèse a priori d'un rapport constant entre flux et stock, ce qui revient à considérer que la dynamique de la population jeune est relativement constante d'une académie à l'autre, d'une part, et que la mobilité entre académie est négligeable, d'autre part. Si la première hypothèse est discutable, la seconde est étayée par la faiblesse de la mobilité géographique des non-diplômés, documentée dans la littérature (Bell \& Bernard, 2016). Dans le tableau 1 figurent ces deux ensembles de données, ainsi que le rapport entre flux et stock (rapport RSIEI/SDRP). Ce dernier chiffre exprime l'importance numérique du repérage SIEI par rapport à la population des sans-diplômes. Ainsi, plus il est élevé, plus cela signifie qu'on identifie (à tort ou à raison) un grand nombre de décrocheurs.

On observe d'assez forts contrastes, difficilement explicables par le différentiel de croissance démographique d'une académie à l'autre. L'académie de Créteil comptabilise un nombre important de jeunes repérés dans SIEI par rapport à ce que renseigne le recensement. À l'inverse, l'académie de Nantes enregistre un effectif relativement faible dans SIEI. Pour autant, cela ne signifie pas que le repérage est meilleur dans l'académie de Créteil. C'est en effet dans cette académie que le taux de "non-décrocheurs » dans l'échantillon interrogé est le plus élevé (dernière colonne). 
Tableau 1. Effectifs de décrocheurs : comparaison SIEl/recensement

\begin{tabular}{|l|c|c|c|c|}
\hline \multicolumn{1}{|c|}{ Académies } & $\begin{array}{c}\text { Effectifs repérés } \\
\text { par SIEI : RSIEI (SIEI } \\
\text { 2014) }\end{array}$ & $\begin{array}{c}\text { Effectifs sans diplôme } \\
\text { autre que le brevet } \\
\text { parmi les 16-24 ans : } \\
\text { SDRP (RP 2012) }\end{array}$ & $\begin{array}{c}\text { Rapport RSIEI/ } \\
\text { SDRP }\end{array}$ & $\begin{array}{c}\text { Part des personnes } \\
\text { déclarant ne pas } \\
\text { avoir interrompu } \\
\text { leurs études au sein } \\
\text { de l'échantillon de } \\
\text { l'enquête MODS 2015 }\end{array}$ \\
\hline Aix-Marseille & 7824 & 44209 & 0,18 & 36,6 \\
\hline Amiens & 5920 & 35654 & 0,17 & 26,1 \\
\hline Bordeaux & 6115 & 41318 & 0,15 & 36,2 \\
\hline Créteil & 13527 & 7114 & 0,19 & 40,9 \\
\hline Nantes & 6125 & 45021 & 0,14 & 39,4 \\
\hline
\end{tabular}

Sigles : SIEl : Système interministériel d'échange d'informations. RSIEI : repérage SIEI ; SDRP : sans diplôme au recensement de la population. Source : INSEE ; MODS 2015, Bernard \& Michaut, CREN.

À ce stade, on ne peut pas aller plus loin dans l'analyse de cette étape, mais elle permet de constater des inégalités territoriales importantes dans le repérage du décrochage scolaire. Ces inégalités renvoient aux fonctionnements institutionnels : informations données par les établissements sur les sorties, échanges entre les réseaux, fiabilité des données académiques variant d'un territoire à l'autre. Un premier constat permet d'établir le caractère déterminant de ces inégalités d'offre institutionnelle sur le recours aux dispositifs d'accompagnement.

\subsection{L'effet de l'académie dans la prise de contact}

La deuxième étape renvoie également aux ressources institutionnelles, du moins en partie. Dans quelle mesure les acteurs de la lutte contre le décrochage contactent-ils réellement les jeunes qu'ils ont repérés?

Une première approche quantitative peut être réalisée à partir des fichiers SIEI. Dans ceux-ci, une variable "Etat » permet de savoir si un jeune a été contacté ou non, entre le moment du recensement et le moment ou ce fichier est consulté. Précisons qu'il s'agit des contacts que doivent réaliser les professionnels dans le cadre des PSAD à partir des listes fournies par SIEI. Concrètement, ce sont souvent des conseillers d'orientation qui se chargent de ce travail. Mais les chiffres indiqués par la base SIEI ne signifient pas que les jeunes "non contactés " ne l'ont pas étés dans un autre cadre.

Toutefois, cela nous semble être un bon indicateur de non-recours, dans la mesure où, comme on l'a vu plus haut, un grand nombre de décrocheurs sont "perdus de vue » une fois sortis de l'école. Dans le cas traité ici, le recensement a été effectué en novembre, pour un fichier arrêté en mars de l'année suivante. Il y a donc potentielle- 
ment cinq mois d'interventions possibles enregistrées dans la base. Sur l'ensemble de l'échantillon étudié ( $\mathrm{N}=7199)$, ce sont $57 \%$ des jeunes qui n'ont pas été contactés.

Ce chiffre varie-t-il sensiblement selon la région où habitent les personnes ? Pour répondre à cette question, il est nécessaire de raisonner " toutes choses égales par ailleurs ». Les populations des cinq académies sont en effet hétérogènes. Notamment, les jeunes en décrochage n'ont pas quitté l'école aux mêmes niveaux (du collège aux classes terminales des lycées), ni dans les mêmes voies de formation (générale, technologique ou professionnelle).

Pour traiter cette hétérogénéité, un modèle logistique a été construit pour tester l'effet de l'académie sur la probabilité de ne pas avoir été contacté. Le choix de l'académie est retenu dans la mesure où la politique de lutte contre le décrochage scolaire est définie au niveau académique, à partir de directives nationales. Ce choix est évidemment discutable, les déclinaisons locales de cette politique académique pouvant varier. Les variables de contrôle sont les caractéristiques individuelles des jeunes figurant dans le fichier : le sexe, l'âge, la durée entre l'interruption de formation et la consultation du fichier, et le dernier niveau de formation atteint. Ces variables sont considérées comme indépendantes entre elles, leurs effets sont donc mesurés " toutes choses égales par ailleurs $»^{3}$.

On trouve un résultat très significatif de l'effet de l'académie sur cette deuxième phase du processus d'accompagnement. Si on prend comme référence l'académie de Nantes, la probabilité relative de ne pas avoir été contacté est plus de deux fois plus élevée dans l'académie d'Aix-Marseille et dans l'académie d'Amiens. On constate également l'importance du niveau de formation dès cette phase, avec une probabilité moins forte d'être contacté quand on décroche du collège ou des classes de seconde et de première de l'enseignement général, par rapport aux décrocheurs de l'enseignement professionnel. Ce résultat original tendrait à montrer une plus forte attention aux situations de décrochage scolaire dans l'enseignement professionnel, ce qui va dans le même sens que les travaux de Khouaja \& Moullet (2016) sur les effets établissements dans la lutte contre le décrochage scolaire.

3. Plusieurs modèles ont été testés, dont certains avec interactions entre variables. Ils ne montrent pas, dans l'ensemble, de résultats significatifs de ces interactions sur les variables dépendantes testées. 
Tableau 2. Modélisation logistique de la probabilité pour un décrocheur de ne pas avoir été contacté dans le cadre des PSAD (rapports de chance)

\begin{tabular}{|l|c|}
\hline Variables & Exp(B) \\
\hline Filles (Référence : garçons) & $\mathrm{n} . \mathrm{s}$ \\
\hline Académie (Référence : académie de Nantes) & 2,555 \\
Aix-Marseille & 2,139 \\
Amiens & 1,827 \\
Bordeaux & 1,746 \\
Créteil & 1,079 \\
\hline Age & 0,999 \\
\hline Durée du décrochage & \\
\hline Niveau de formation au moment du décrochage (Réf : Niveau V professionnel) & 1,441 \\
Collège & 1,869 \\
Niveau V général et technologique & 0,741 \\
MLDS et autres & $1,197^{*}$ \\
\hline Niveau IV général et technologique & $n . s$ \\
Niveau IV professionnel & $n .5$ \\
CAP 1ère année & 0,057 \\
\hline Pseudo R2 (Nagelkerke) & \\
\hline
\end{tabular}

Note : Les valeurs sont des rapports de chance, appelées également probabilités relatives. Par exemple, la probabilité pour un jeune de ne pas avoir été contacté plutôt que d'avoir été contacté est 1,798 fois plus élevée dans l'académie d'Aix-Marseille que dans l'académie de Nantes. Un rapport de chance supérieur à 1 traduit un effet positif de la variable considérée sur la variable dépendante, ici le fait de ne pas avoir été contacté, un rapport de chance inférieur à 1 indique un effet négatif. Les valeurs indiquées sont significatives au seuil de $5 \%$. Les effets au seuil de $10 \%$ sont indiqués par une étoile. Au-dessus, les valeurs ne sont pas indiquées (n.s: non significatif).

Source : MODS 2015, Bernard \& Michaut, CREN.

Une autre façon d'appréhender la question de la prise de contact est de se référer à ce qu'en disent les jeunes eux-mêmes, et non ce qui est inscrit dans les bases de données. L'enquête MODS ( $\mathrm{N}=2948)$ fournit une donnée particulièrement pertinente dans cette perspective. À la question : "Depuis que vous avez arrêté l'école, avez-vous rencontré l'une des personnes suivantes ", suivie d'une liste de professionnels susceptibles de réaliser un accompagnement, les jeunes pouvaient répondre "aucune de ces personnes". L'indicateur retenu ici pour la phase de "prise de contact " est plus restrictif que dans le cas précédent, puisque la question porte sur l'éventualité d'une rencontre, ce qui exclut par exemple le simple contact téléphonique. Par ailleurs, cet indicateur laisse plus de place à la logique d'action du jeune. Ainsi, celui-ci peut, notamment, ne pas avoir cherché à rencontrer un professionnel de l'accompagnement s'il estime qu'il n'en a pas besoin, par exemple s'il occupe un emploi.

On a donc testé l'effet de l'académie sur la probabilité de n'avoir rencontré aucun professionnel de l'accompagnement depuis le décrochage. Cette situation concerne 
23,7 \% de l'échantillon ${ }^{4}$. Comme dans le cas précédent, les variables retenues sont les caractéristiques individuelles des décrocheurs : sexe, âge, ancienneté du décrochage, dernier niveau de formation atteint. Ont été ajoutées une variable de milieu social, à travers la catégorie socioprofessionnelle du père, et une variable de situation au moment de l'enquête. On peut en effet supposer que les jeunes ayant décroché pour exercer un emploi, par exemple, n'ont pas été contactés pour un accompagnement.

De fait, le modèle indique un effet très significatif de la situation au moment de l'enquête sur la probabilité relative d'avoir rencontré un professionnel, puisque le fait d'être en formation multiplie par deux cette probabilité par rapport aux jeunes en emploi. Le milieu social a peu d'effet, si ce n'est que les enfants d'indépendants ont moins de probabilité d'avoir rencontré de professionnels que les autres. On peut voir là encore l'effet de ressources alternatives à l'accompagnement quand les parents peuvent offrir à leurs enfants des perspectives liées à un milieu professionnel d'indépendants. Le niveau de formation a également un effet, les jeunes ayant décroché en collège ou dans les deux premières années du lycée général et technologique déclarant plus souvent ne pas avoir rencontré de professionnel. Symétriquement, les jeunes ayant interrompu leur formation au niveau $\mathrm{V}^{5}$ des filières professionnelles déclarent plus souvent avoir rencontré des professionnels. La prise en compte des variables de contrôle permet de mettre en évidence un effet encore significatif de l'académie sur cette probabilité de rencontrer ou non des professionnels de l'accompagnement. Ainsi, les jeunes des académies d'Amiens, de Bordeaux et de Créteil déclarent de manière très significative moins d'accompagnement que les jeunes de l'académie de Nantes.

4. Données redressées en fonction de la répartition par sexe et par académie de l'ensemble de la population de référence.

5. Niveau V professionnel : 2ème année de CAP, 2de et 1ère de bac pro. 
Tableau 3. Probabilité d'avoir rencontré un professionnel pour les jeunes en situation de décrochage

\begin{tabular}{|c|c|}
\hline & $\operatorname{Exp}(B)$ \\
\hline Filles (Référence : garçons) & ns \\
\hline \multicolumn{2}{|c|}{ Académie (Référence : académie de Nantes) } \\
\hline Aix-Marseille & $0,744^{*}$ \\
\hline Amiens & 0,682 \\
\hline Bordeaux & 0,683 \\
\hline Créteil & 0,703 \\
\hline Age & ns \\
\hline Durée du décrochage & 1,059 \\
\hline \multicolumn{2}{|c|}{ Niveau de formation au moment du décrochage (Réf : Niveau V professionnel) } \\
\hline Collège & 0,554 \\
\hline Niveau V général et technologique & 0,512 \\
\hline Niveau IV général et technologique & $0,744^{*}$ \\
\hline MLDS et autres & 1,749 \\
\hline CAP $1^{\text {ère }}$ année & ns \\
\hline Niveau IV professionnel & ns \\
\hline \multicolumn{2}{|l|}{ PCS du père (Réf: Ouvriers) } \\
\hline Indépendants & ,681 \\
\hline Cadres et professions intermédiaires & ns \\
\hline Employés & ns \\
\hline \multicolumn{2}{|l|}{ Situation actuelle (Réf. : inactif) } \\
\hline Emploi & ns \\
\hline Chômage & 2,583 \\
\hline Formation & 1,988 \\
\hline Pseudo R2 (Nagelkerke) & 0,107 \\
\hline
\end{tabular}

Note : Les valeurs sont des rapports de chance, appelées également probabilités relatives. Un rapport de chance supérieur à 1 traduit un effet positif de la variable considérée sur la variable dépendante, ici le fait d'avoir rencontré un professionnel, un rapport de chance inférieur à 1 indique un effet négatif. Les valeurs indiquées sont significatives au seuil de $5 \%$. Les effets au seuil de $10 \%$ sont indiqués par une étoile. Au-dessus, les valeurs ne sont pas indiquées (n.s: non significatif).

Source : MODS 2015, Bernard \& Michaut, CREN. 


\subsection{Les propositions d'aide varient selon le type de professionnel rencontré}

Une fois le contact établi, les jeunes peuvent se voir proposer une aide dans un domaine particulier (reprise de formation, accès à la qualification professionnelle, accompagnement vers l'emploi, recherche de logement, accès aux soins, etc.). Comme vu plus haut, il s'agit bien d'une étape spécifique. Elle s'inscrit d'abord dans une interaction dont la perception réciproque par les acteurs est déterminante.

L'enquête MODS offre la possibilité d'étudier les facteurs de cette étape à partir de la question : "L'une des personnes précédentes vous a-t-elle proposé", suivie d'une série de propositions, parmi lesquelles « aucune proposition d'aide». Cette modalité est choisie à ce niveau comme variable dépendante, et l'effet de l'académie est testé avec les variables de contrôle indiquées précédemment. Sur l'échantillon de personnes interrogées et qui ont effectivement rencontré un professionnel $(\mathrm{N}=2190), 15,4 \%$ déclarent ne pas avoir reçu de proposition d'aide.

À ce stade, l'académie n'a pas d'effets significatifs. La situation au moment de l'enquête affecte encore fortement la probabilité de s'être vu proposer une aide, dans le même sens que précédemment (les jeunes en emploi déclarent moins souvent avoir obtenu une aide que les jeunes au chômage ou en formation). Quand on introduit dans le modèle les types de personnel rencontrés, certains d'entre eux ont un effet très significatif. L'accompagnement par un éducateur spécialisé augmente ainsi très fortement la probabilité relative de s'être vu proposer une aide, à l'inverse de l'accompagnement d'un conseiller Pôle emploi ou, dans une moindre mesure, un conseiller Mission locale. On peut y voir l'effet de formes plus personnalisées d'accompagnement, à l'opposé de pratiques plus institutionnelles et standardisées. 
Tableau 4. Probabilité d'avoir eu une proposition d'aide

\begin{tabular}{|c|c|}
\hline & $\operatorname{Exp}(B)$ \\
\hline Filles (Référence : garçons) & ns \\
\hline \multicolumn{2}{|c|}{ Académie (Référence : académie de Nantes) } \\
\hline Aix-Marseille & ns \\
\hline Amiens & ns \\
\hline Bordeaux & ns \\
\hline Créteil & ns \\
\hline Age & ns \\
\hline Durée du décrochage & ns \\
\hline \multicolumn{2}{|c|}{$\begin{array}{l}\text { Niveau de formation au moment du décrochage (Réf: Niveau V } \\
\text { professionnel) }\end{array}$} \\
\hline Collège & ns \\
\hline Niveau $V$ général et technologique & ns \\
\hline Niveau IV général et technologique & ns \\
\hline MLDS et autres & 0,616 \\
\hline CAP 1ère année & $0,604^{*}$ \\
\hline Niveau IV professionnel & ns \\
\hline \multicolumn{2}{|l|}{ PCS du père (Réf: Ouvriers) } \\
\hline Indépendants & 0,663 \\
\hline Cadres et professions intermédiaires & 0,638 \\
\hline Employés & 0,698 \\
\hline \multicolumn{2}{|l|}{ Situation actuelle (Réf. : inactif) } \\
\hline Emploi & ns \\
\hline Chômage & 1,468 \\
\hline Formation & 2,258 \\
\hline \multicolumn{2}{|l|}{ Professionnels rencontrés (Réf: non) } \\
\hline Conseiller d'orientation & ns \\
\hline Proviseur ou principal & ns \\
\hline Personne de la MLDS & ns \\
\hline Conseiller Mission locale & 0,746 \\
\hline Conseiller Pôle emploi & 0,331 \\
\hline Assistante sociale & ns \\
\hline Éducateur spécialisé & 6,261 \\
\hline Pseudo R2 (Nagelkerke) & 0,108 \\
\hline
\end{tabular}

Note : Les valeurs sont des rapports de chance, appelées également probabilités relatives. Un rapport de chance supérieur à 1 traduit un effet positif de la variable considérée sur la variable dépendante, ici le fait d'avoir eu une proposition d'aide, un rapport de chance inférieur à 1 indique un effet négatif. Les valeurs indiquées sont significatives au seuil de $5 \%$. Les effets au seuil de $10 \%$ sont indiqués par une étoile. Au-dessus, les valeurs ne sont pas indiquées (ns: non significatif)

Source : MODS 2015, Bernard \& Michaut, CREN. 


\subsection{L'enrôlement dépend du professionnel rencontré et de la nature de l'aide proposée}

Avoir une proposition d'aide ne signifie pas pour autant qu'on l'accepte ou qu'on la suit. Sur les 1722 personnes ayant reçu une proposition d'aide après leur décrochage, $15,3 \%$ ne l'ont pas suivie pendant la période comprise entre leur décrochage et l'enquête (en moyenne un an). À partir d'une nouvelle modélisation logistique, on observe à nouveau la prééminence des interrelations entre jeunes et professionnels sur les autres variables pour expliquer l'enrôlement. Les différences sont très sensibles selon les professionnels rencontrés. Les conseillers MLDS sont ceux qui renforcent le plus l'engagement des jeunes dans un parcours d'accompagnement. À l'opposé, les propositions faites par les chefs d'établissements scolaires sont celles qui se traduisent le plus souvent par des refus, ce qui révèle bien le rapport difficile qu'entretiennent les jeunes en situation de décrochage avec leurs établissements d'origine. L'enrôlement dépend également des propositions faites. C'est quand ils reçoivent des propositions d'aide financière, de stage ou de place dans un organisme de formation, soit des ressources visant à un raccrochage en formation, que les jeunes s'enrôlent le plus dans un parcours d'accompagnement.

Tableau 5. Probabilité de ne pas accepter l'aide proposée

\begin{tabular}{|c|c|}
\hline & $\operatorname{Exp}(B)$ \\
\hline Filles (Référence : garçons) & ns \\
\hline \multicolumn{2}{|c|}{ Académie (Référence : académie de Nantes) } \\
\hline Aix-Marseille & ns \\
\hline Amiens & ns \\
\hline Bordeaux & $0,647^{*}$ \\
\hline Créteil & ns \\
\hline Age & ns \\
\hline Durée du décrochage & ns \\
\hline \multicolumn{2}{|c|}{$\begin{array}{l}\text { Niveau de formation au moment du décrochage (Réf : Niveau V } \\
\text { professionnel) }\end{array}$} \\
\hline Collège & ns \\
\hline Niveau V général et technologique & $0,487^{*}$ \\
\hline Niveau IV général et technologique & ns \\
\hline MLDS et autres & ns \\
\hline CAP 1ère année & ns \\
\hline Niveau IV professionnel & ns \\
\hline \multicolumn{2}{|l|}{ PCS du père (Réf: Ouvriers) } \\
\hline Indépendants & ns \\
\hline Cadres et professions intermédiaires & ns \\
\hline Employés & $0,723^{*}$ \\
\hline
\end{tabular}




\begin{tabular}{|c|c|}
\hline \multicolumn{2}{|l|}{ Situation actuelle (Réf. : inactif) } \\
\hline Emploi & ns \\
\hline Chômage & ns \\
\hline Formation & 0,527 \\
\hline \multicolumn{2}{|l|}{ Professionnels rencontrés (Réf : non) } \\
\hline Conseiller d'orientation & ns \\
\hline Proviseur ou principal & 3,248 \\
\hline Personne de la MLDS & 0,458 \\
\hline Conseiller Mission locale & ns \\
\hline Conseiller Pôle emploi & ns \\
\hline Assistante sociale & ns \\
\hline Educateur spécialisé & ns \\
\hline \multicolumn{2}{|l|}{ Aide proposée (Réf: non) } \\
\hline Une place dans un établissement de formation & 0,465 \\
\hline Une offre d'emploi & ns \\
\hline Une offre de stage & 0,548 \\
\hline Un accompagnement vers l'emploi & 0,474 \\
\hline Un accompagnement vers la formation & 0,649 \\
\hline Une aide financière & 0,251 \\
\hline Un hébergement & ns \\
\hline Pseudo R2 (Nagelkerke) & 0,146 \\
\hline
\end{tabular}

Note : Les valeurs sont des rapports de chance, appelées également probabilités relatives. Un rapport de chance supérieur à 1 traduit un effet positif de la variable considérée sur la variable dépendante, ici le fait de ne pas avoir accepté l'aide proposée, un rapport de chance inférieur à 1 indique un effet négatif. Les valeurs indiquées sont significatives au seuil de $5 \%$. Les effets au seuil de $10 \%$ sont indiqués par une étoile. Au-dessus, les valeurs ne sont pas indiquées (ns : non significatif).

Source : MODS 2015, Bernard \& Michaut, CREN.

\section{Conclusion}

Trois points peuvent être retenus au terme de cette analyse. Tout d'abord, elle met en évidence le caractère déterminant des premières phases du processus qui conduit un jeune en décrochage scolaire à être accompagné ou non pour accéder à une formation ou à l'emploi. Ces premières phases créent une véritable sélection des individus permettant d'expliquer l'importance du non-recours par non-connaissance du dispositif (au sens de Warin, op. cit.). L'étude apporte ainsi des résultats originaux sur cette question du repérage et de ses conséquences en matière d'accompagnement. De nombreux jeunes en décrochage scolaire ne bénéficient pas de l'offre de remédiation institutionnelle, soit parce qu'ils ne sont pas repérés comme tels, soit parce qu'ils n'ont fait l'objet d'aucune prise de contact par des professionnels, bien qu'ils aient été repérés comme décrocheurs. Ce non-recours est d'autant plus problématique qu'il est inégal selon les territoires. Il y a là un premier levier d'action publique pour une meilleure prise en charge des personnes les plus en difficulté.

Le deuxième point qui mérite d'être souligné est le poids toujours important des déterminants scolaires dans le recours à la remédiation, et ce essentiellement encore une fois 
dans les premières séquences du recours. Que ce soit au niveau des prises de contact ou des rapports avec les professionnels, les décrocheurs du collège ont moins recours à l'offre publique de remédiation que les autres. Or, ce sont les élèves qui ont connu le plus de difficultés durant leur scolarité, d'où d'ailleurs leur décrochage précoce. Les institutions en charge de la lutte contre le décrochage rencontrent visiblement des difficultés à contacter et à rencontrer les jeunes les plus fragiles. On retrouve ici le caractère sélectif de nombreux dispositifs des politiques publiques envers les personnes les plus fragiles.

Enfin, le dernier point concerne l'importance des interactions qui se jouent entre jeunes et professionnels. Certes, la méthodologie quantitative utilisée ici ne permet pas d'accéder à ces interactions. Mais elle montre que plus on avance dans ce processus séquentiel, moins les variables caractérisant les personnes ou les contextes territoriaux (ici les académies) ont d'effet. Elle met plutôt en évidence des inégalités d'effets de raccrochage selon les professionnels et les propositions d'aides, ce qui confirme les analyses qualitatives sur les interactions entre professionnels et publics (Rouleau-Berger, op. cit. ; Denecheau \& al., op. cit.). Ce sont les personnels de l'éducation spécialisée et des Missions de lutte contre le décrochage scolaire qui enrôlent le mieux les jeunes dans un parcours d'accompagnement. Et ce sont les propositions les plus concrètes (aides financières, stages et propositions de formation) qui permettent de recueillir l'assentiment des bénéficiaires, et d'éviter ainsi un deuxième " décrochage ".

\section{Bibliographie}

Bell L., Bernard P.-Y. (2016), «Territoires, offre de formation et expérience du décrochage scolaire : une étude de cas ", Espaces et Sociétés, n 166(3), pp. 5-11.

Benhaim-Grosse J. (2012), "Les pratiques éducatives et pédagogiques des enseignants auprès des élèves accueillis en dispositifs relais l'année 2009-2010 ", Note d'informationDEPP, n ${ }^{\circ} 12.14$.

Bernard P.-Y. (2009), La politique de traitement du décrochage scolaire. Le cas de la Mission générale d'insertion de l'Éducation nationale, Thèse de doctorat en sociologie, sous la direction de Yves Dutercq, Université de Nantes.

Bernard P.-Y. (2015), Le décrochage scolaire, Paris, PUF, 128 p.

Bernard P.-Y., Michaut C. (2006), « La Mission Générale d'Insertion de l'Education nationale : des publics hétérogènes, une mission d'insertion? ", Recherches en Éducation, $\mathrm{n}^{\circ} 1$, pp. 43-50.

Bernard P.-Y., Michaut C. (2013), “The effects of the fight against early school- leaving: Back to education or school-to-work transition?”, in De Groof S. \& Elchardus M. (eds), Early school-leaving and Youth unemployment, Leuven: Lannoo Campus Press, pp. 132-156. 
Bernard P.-Y., Michaut C. (2014a), « Le partenariat interinstitutionnel : un nouvel instrument de politique éducative ? Le cas des politiques de traitement du décrochage scolaire ", Education comparée, ${ }^{\circ} 11$, pp. 111-131.

Bernard P.-Y., Michaut C. (2014b), “"Marre de l'école”. Une analyse des motifs de décrochage scolaire ", Note du CREN n 17.

Bernard P.-Y., Michaut C. (2016), "Les motifs de décrochage par les élèves. Un révélateur de leur expérience scolaire ", Education \& formations, n 90, pp. 95-112.

Berthet T., Zaffran J. (2014), Le décrochage scolaire. Enjeux, acteurs et politiques de lutte contre la déscolarisation, Presses universitaires de Rennes, Rennes, 185 p.

Castra D. (2006), Linsertion professionnelle des publics précaires, Paris, PUF, 243 p.

Darmon I., Frade C., Demazière D., Haas I (2004), "Formés et formateurs face à la double contrainte des programmes de formation à l'employabilité des chômeurs de longue durée ", Formation Emploi, n 85, pp. 57-75

De Foucauld J.-B. (dir.) (2010), Les missions locales et l'insertion professionnelle et sociale des jeunes, Rapport pour l'inspection générale des finances, Paris, La Documentation française.

Denantes J. (2008), "Échouez d'abord, on s'occupera de vous ensuite ", Actualité de la formation permanente, $\mathrm{n}^{\circ} 210$, pp. 55-61.

Denecheau B., Houdeville G., Mazaud C. (2015), À l'école de l'autonomie. Épreuves et enjeux des dispositifs de deuxième chance, Paris, L'Harmattan, 280 p.

Demailly L. (2009), "Fortunes et ambiguïtés de l'accompagnement », EMPAN, n 74(2), pp. 21-28.

Dmitrijeva J., Fremigacci F., L'Horty Y. (2015), « Le paradoxe des nouvelles politiques d'insertion ", Revue d'économie politique, n 125(4), pp. 475-498.

Eurydice-Cedefop (2014), Tackling early leaving from education and training in Europe. Measures and strategies, Eurydice report.

Heckman J., Smith J. (2003) "The Determinants of Participation in a Social Program: Evidence from a Prototypical Job Training Program”, IZA Discussion paper series, $\mathrm{n}^{\circ} 798$.

Khouaja E. \& Moullet S. (2016), "Le rôle des caractéristiques des établissements dans le décrochage scolaire : l'exemple de l'académie d'Aix-Marseille ", Formation Emploi, $\mathrm{n}^{\circ} 134$, pp. 7-26.

Mas S. (2004), «Un jeune sur deux en emploi à la sortie de TRACE », Premières synthèses, DARES, $\mathrm{n}^{\circ} 34.1$.

Paul M. (2009), "L'accompagnement dans le champ professionnel ", Savoirs, n 20(2), pp. 11-63. 
Plessard C., Simon V., Berthet T. (2014), Etude du temps de latence chez les jeunes de 16 à 25 ans inscrits à la Mission locale en Rhône-Alpes, Céreq, université de Bordeaux.

PRAO (2013), Mission d'observation du raccrochage en formation et en faveur de l'emploi. Synthèse et analyse statistique.

Rouleau-Berger L. (1998) "Professionnels de l'insertion au contact des jeunes en situation précaire : de la coproduction et de la discrimination des compétences ", Lien social et Politiques, $\mathrm{n}^{\circ}$ 40, pp. 39-45.

Warin P. (2003), «Le non-recours aux services publics, une question en attente de reconnaissance ", Informations sociales, $n^{\circ} 109$, pp. 94-101.

Warin P. (2012), «Le non-recours aux droits », SociologieS [En ligne], Théories et recherches, URL : http://sociologies.revues.org/4103.

White J., Wehlage G. (1995), “Community Collaboration: If It Is Such a Good Idea, Why Is It So Hard to Do?”, Educational Evaluation and Policy Analysis, n 17(1), pp. 23-38.

Zunigo X. (2008), "L'apprentissage des possibles professionnels. Logiques et effets sociaux (des Missions locales pour l'emploi des jeunes) ", Sociétés contemporaines, $\mathrm{n}^{\circ} 70$, pp. 115-132. 